Distribution Categories:

Plasma Systems (UC-20a)

Fusion Systems (UC-20d)

ANL/FPP/TM-116

ARGONNE NATIONAL LABORATORY

9700 South Cass Avenue

Argonne, Illinois 60439

ON NEOCLASSICAL TRANSPORT IN PLASMAS CONTAINING IONS WITH MULTIPLE CHARGE STATES

by

C. D. Boley

Applied Physics Division

This report uas prepared as at account of work sponsored by the bnited States Government. Neither the

Uniled States not the United States Department of

Enerisy, not any of their empluyees. nor any of their

contracturs, subcontractors, or their empioyecs. makes

any warranty, express or implicd, or assumes any legal

liability or tespunsibility for the' accuracy, completeness

or usefulness of any informationt, apparatus. product of

process fisclosed. or represents that its ute woutd net

infringe privately owned rights.

October 1978 


\title{
ON NEOCLASSICAL TRANSPORT IN PLASMAS CONTAINING IONS WITH MULTIPLE CHARGE STATES
}

by

\author{
C. D. Boley \\ Aplited Physias Division \\ Argonic National Laboratory \\ Argonne, Illinois oot33
}

\begin{abstract}
It is shown that, in the calculation of the Spitzer function and the Pfirsch-Schlüter transport coefficients of a multispecies plasma, a considerable simplication occurs for the case of ions with multiple charge states. The number of coupled integral equations to be solved can be recuced from the total number of species to the number of species with different nuclei. This generalizes an earlier treatment, in which such a reduction was found under the assumption of the disparate mass approximation. The solutions are expressed in terms of quantities defined wholly within a system of simple ions.
\end{abstract}




\section{INTRODUCTION}

In a recent report, some applications of the neoclassical transport theory of a multispecies tokamak plasma were worked out. $(1,2)$ The system consisted of electrons and an arbitrary number of ion species, each possibly containing a number of charge states. In addition, for computational reasons, the "disparate mass" approximation was employed, according to which the masses of the ions satisfied $m_{1} \ll m_{2} \ll \ldots . m_{N}$. Two calculations were presented - one of the multispecies Spitzer function (response of the distribution function to a parallel electric field), and the other of the multispecies transport coefficients in the Pfirsch-Schlüter regime. As was noted, the importance of the Spitzer function lies in the fact that it is required in the evaluation of the transport coefficients associated with the Ware pinch and the bootstrap current. Interest in the detailed behavior of a complex ion system (preferably, of course, without the disparate mass approximation) arises from the marked presence of impurities in many tokamak experiments.

In the present report it is shown that many of the results of Ref. 1 , such as the explicit dependence of the solutions on the charge-state indices, are actually independent of the disparate mass approximation. They follow quite generally from the scaling properties of the Coulomb collision kernel.

In connection with this, it will be shown that for purposes of calculating the Spitzer function and the multispecies Pfirsch-Schlüter transport coefficients, one need not consider the dynamics of individual charge states. Instead one can always reduce the problem to the dynamics of a reduced system which contains only $N+1$ constituents, where $N$ is the number of ion species with different nuclei. These constituents have effective ‘ensities and charges which are given by appropriate averages over the charge states. Thus, for example, in a system 
of electrons, protons, oxygen, and tungsten, the number of coupled equations to be solved can be reduced from 84 to 4 . This simplification would appear to be very useful in practical computations.

Following the conventional formulation of plasma transport, atomic processes such as ionization and recombination are taken into account not in the kinetic theory but in the ensuing diffusion theory. Thus the kinetic equation involves only Coulomb collisions.

\section{SPITZER FUNCTION}

Whenever possible we use the notation of Ref. 1. The various ion species are labeled by $a, b$, etc., and the charge states by $i, j$. (The terminology "ion species" refers to all ions with the same nucleus.) Thus the density and charge of charge state $\underline{i}$ within ion species $\underline{a}$ are, respectively, $n_{a i}$ and $e_{a i} \cdot$ All charge states within ion species a are assumed to be characterized by a common temperature $T_{a}$. Of course they have the same mass $m_{a}$. Since only Coulomb collisions are considered, it is permissible to treat the electrons as one of the "ion species".

The version of the Landau-Vlasov kinetic equation which occurs in the Spitzer problem ${ }^{(3)}$ and in the Pfirsch-Schlüter transport problem ${ }^{(4)}$ has the time derivative removed, the collision term linearized about a local Maxwellian without drift, and the remaining terms operating on this local Maxwellian. The densities and temperatures ary only in the direction parallel to the local magnetic field, and the (given) electric field is in the parallel direction. Thus there are three driving terms. In the Spitzer problem only the driving term containing the electric field is considered. We therefore have 


$$
\sum_{b j}\left\{c_{a i, b j}\left[f_{a i}^{(1)}, f_{b j}^{(0)}\right]+c_{a i, b j}\left[f_{a i}^{(0)}, f_{b j}^{(1)}\right]\right\}=-\frac{e_{a i} E_{n} v_{1}}{T_{a}} f_{a i}^{(0)},
$$

where $C_{a 1, b j}$ is the linearized Coulomb collision operator and the solution has been written as $f_{a i}=f_{a i}^{(0)}+f_{a i}^{(1)}$, with

$$
\begin{aligned}
f_{\mathrm{ai}}^{(0)}(v) & =n_{\mathrm{a}{ }^{\phi} \mathrm{a}}(v), \\
\phi_{\mathrm{a}}(v) & =\left(\pi v_{\mathrm{Ta}}^{2}\right)^{-3 / 2} \exp \left(-v^{2} / v_{\mathrm{Ta}}^{2}\right), \quad v_{\mathrm{Ta}}^{2}=2 \mathrm{~T}_{\mathrm{a}} / \mathrm{m}_{\mathrm{a}}
\end{aligned}
$$

The solvability condition of Eq. (1) imposes local charge neutrality: $\sum_{a i} n_{a i} e_{a i}=0$.

To bring out the charge-state dependence in Eq. (1) we first write the two parts of the collision operator in terms of the appropriate collision kernels:

$$
\begin{aligned}
& c_{a i, b j}\left[f_{a i}^{(1)}, f_{b j}^{(0)}\right]=\int d^{3} v^{-} J_{a i, b j}\left(\vec{v}, \vec{v}^{-}\right) h_{a i}\left(\vec{v}^{-}\right), \\
& c_{a i, b j}\left[f_{a i}^{(0)}, f_{b j}^{(1)}\right]=\int d^{3} v^{-} k_{a i, b j}\left(\vec{v}, \vec{v}^{-}\right) h_{b_{j}}\left(\vec{v}^{-}\right),
\end{aligned}
$$

where the perturbation has been written $f_{a i}^{(1)}\left(\vec{v} ;=n_{a i}{ }_{a}(v) h_{a i}(\vec{v})\right.$. As Eq. (3) indicates, $J$ is the kernel of the test-particle part of the collision operator and $K$ is the kernel of the field-particle part. Now because the Coulomb collision operator is taken from weak-coupling theory, the individual charges enter the kernels only as an overall factor $e_{a i}^{2} e_{b j}^{2}$. Similarly, the densities enter as an overall factor $n_{a i} n_{b j}$. (The cutoff factor $n \Lambda$ depends on the charges and densities but, of course, is independent of the indices on the kernels.) Thus the kerneis have the form 


$$
\begin{aligned}
& J_{a i, b j}\left(\vec{v}, \vec{v}^{-}\right)=\xi_{a i} \xi_{b j} J_{a b}\left(\vec{v}, \vec{v}^{-}\right), \\
& K_{a i, b j}\left(\vec{v}, \vec{v}^{-}\right)=\xi_{a i} \xi_{b j} K_{a b}\left(\vec{v}, \vec{v}^{-}\right),
\end{aligned}
$$

where

$$
\xi_{a i}=n_{a i} e_{a i}^{2}\left(\sum_{j} n_{a j} e_{a j}^{2}\right)-1
$$

with the normalization factor in $\xi_{\text {ai }}$ a matter of convenience. The decomposition in Eqs. (4) fails if not all charge states associated with a given ion species have the same temperature.

Making these substitutions in Eq. (1), we have the system

$$
\xi_{a i} \int d^{3} v^{-}\left[J_{a}\left(\vec{v}, \vec{v}^{-}\right) h_{a i}\left(\vec{v}^{-}\right)+\sum_{b} k_{a b}\left(\vec{v}^{\prime}, \vec{v}^{-}\right) \bar{h}_{b}\left(\vec{v}^{-}\right)\right]=-n_{a i} e_{a \dot{i}} \phi_{a}(v) \frac{E_{\|} v_{u}}{T_{a}},
$$

where $J_{a}=\sum_{b} J_{a b}$ and $\bar{h}_{a}$ is the solution averaged over the charge states of the ion species:

$$
\bar{h}_{a}(\vec{v})=\sum_{i} \xi_{a i} h_{a i}(\vec{v})
$$

Two elementary but important results can now be inferred from Eg. (6). First, summing over $\underline{i}$, we obtain a closed equation for the $\bar{h}$ 's:

$$
\sum_{b} \int d^{3} v^{-}\left[\delta_{a b} J_{a}\left(\vec{v}, \vec{v}^{-}\right)+k_{a b}\left(\vec{v}, \vec{v}^{-}\right)\right] \bar{h}_{b}\left(\vec{v}^{-}\right)=-\left(\sum_{i} n_{a i} e_{a i}\right) \phi_{a}(v) \frac{E_{a} v_{y}}{T_{a}} .
$$

This is just the Spitzer problem for a modified plasma in which all charge states of a given ion species in the original plasma are replaced by a single constituent of mass $m_{a}$, with a density $\bar{n}_{a}$ and a charge $\bar{e}_{a}$ given by 


$$
\vec{n}_{a}=\frac{\left(\sum_{i} n_{a i} e_{a i}\right)^{2}}{\sum_{i} n_{a i} e_{a i}^{2}}, \quad \bar{e}_{a}=\frac{\sum_{i} n_{a i} e_{a i}^{2}}{\sum_{i} n_{a i} e_{a 1}} .
$$

The solution $\vec{h}_{a}$ is determined by consideration of this reduced system. Second, combining Eq. (8) with Eq. (6), we see that the difference $h_{a i}-\bar{h}_{a}$ satisfies the test-particle equation:

$\int d^{3} v^{-} J_{a}\left(\vec{v}, \vec{v}^{-}\right)\left[h_{a i}\left(\vec{v}^{-}\right)-\bar{h}_{a}\left(\vec{v}^{-}\right)\right]=-\left(\frac{1}{e_{a i}}-\frac{1}{\bar{e}_{a}}\right) \frac{\bar{n}_{a} \tilde{e}_{a}^{2}}{T_{a}} E_{\|} v_{\|} \phi_{a}(v)$.

The salient feature of this equation is the fact that the charge $e_{a i}$ appears separately in the inhomogeneous term but not in the kernel. As in the case for $\bar{h}_{a}$, therefore, this equation is solved by consideration of a system of electrons and reduced ions, with densicies and charges as in Eq. (9).

Since the inhomogeneous term in Eq. (10) has the angular dependence $\cos \theta$, with $\theta$ the polar angle of $\vec{v}$ with respect to the magnetic field, and since the kernel is invariant under rotations, the solution also varies as $\cos \theta$. Thus we can write it as

$$
h_{a i}(\vec{v})=\bar{h}_{a}(\vec{v})+\left(\frac{1}{e_{a i}}-\frac{1}{\bar{e}_{a}}\right) \frac{\bar{e}_{a}^{2}}{\nu_{a a} T_{a}} E_{\|} v_{n} F_{a}\left(t_{a}\right),
$$

where $t_{a}=v^{2} / v_{T_{a}}^{2}$. Multiplying Eq. (10) by $\cos \theta$ and integrating over the solid angle, we find that $F_{a}$ is the (unique) solution of the one-dimensional equation

$$
\int_{0}^{\infty} d t^{*} \sqrt{t^{-}} j_{a}\left(t, t^{-}\right) F_{a}\left(t^{-}\right)=-\frac{2 \bar{n}_{a} \nu_{a a}}{\pi^{3 / 2} v_{T a}^{6}} t e^{-t},
$$


with kerne1

$$
j_{a}\left(t, t^{-}\right)=\frac{1}{4 \pi v_{T a}^{2}} \int d \Omega d \Omega^{-} J_{a}\left(\vec{v}, \vec{v}^{-}\right) \vec{v} \cdot \vec{v}^{*} .
$$

The subscript $\underline{a}$ is implicit on $t$ and $t *$. Although the definition of the collision frequency $v_{a a}$ is immaterial, for purposes of comparison with Ref. 1 we shall take it to be the Braginskij self-collision frequency, multiplied by $\sqrt{2}$ :

$$
\nu_{a a}=\frac{4 \sqrt{2 \pi} \bar{n}_{a} \bar{e}_{a}^{-4} \ln \Lambda}{3\left(4 \pi \varepsilon_{0}\right)^{2}\left(m_{a} T_{a}^{3}\right)^{1 / 2}}
$$

Equation (11) is the main result of this section. According to it, the Spitzer function of any given charge state is the sum of two contributions: the Spitzer function of that ion species in a reduced ion system with densities $\bar{n}_{a}$ and charges $\bar{e}_{a}$, and the solution of the test-particle equation for this reduced system. One is not required to solve a kinetic equation involving the individual dynamics of the charge states. In particular, the combination $\left(e_{a i}^{-1}-\bar{e}_{a}^{-1}\right)^{-1}\left(h_{a i}-\bar{h}_{a}\right)$ is independent of the charge state.

In Ref. 1 a solution having the form of Eq. (11) was obtained within the context of the disparate mass approximation. To recover $i t$, we approximate $\bar{h}_{a}$ and $F_{a}$ by a truncated expansion in Laguerre polynomials,

$$
\begin{aligned}
& \bar{h}_{a}(\vec{v})=\frac{2 v_{\|}}{v_{T a}^{2}} \sum_{k=0}^{k_{\max }} L_{k}^{(3 / 2)}\left(t_{a}\right) \bar{u}_{a}^{k}, \\
& F_{a}(t)=\sum_{k=0}^{k_{\max }} L_{k}^{(3 / 2)}(t) F_{a}^{k},
\end{aligned}
$$

and evaluate $\bar{u}_{a}^{k}$ and $F_{a}^{k}$ in the disparate mass limit. 
In this limit the correspondence is

$$
\begin{aligned}
& \bar{u}_{a}^{k}=\delta_{k 0} w+U_{a}^{k}, \\
& F_{a}^{k}=\left[D^{-1}\left(z_{a}\right)\right]^{k 0},
\end{aligned}
$$

with the/right-hand side in the notation of Ref. 1.

III. DIFFUSION IN THE PFIRSCH-SCHLÜTER REGIME

Turning to the diffusion problem, we consider the kinetic equation with driving terms containing parallel pressure and temperature gradients:

$$
\begin{aligned}
\sum_{b j}\left\{c_{a i, b j}\left[f_{a i}^{(1)}, f_{b j}^{(0)}\right]+c_{a i, b j}\left[f_{a i}^{(0)}, f_{b j}^{(1)}\right]\right\} \\
=\left[\nabla_{\|} \text {ln } p_{a i}-\left(\frac{5}{2}-t_{a}\right) \nabla_{\|} \text {ln } T_{a}\right] v_{\|} f_{a i}^{(0)} .
\end{aligned}
$$

We evaluate first the classical friction coefficients, then the Pfirsch-Schluter radial transport coefficients. ${ }^{(5)}$

As in the previous section, we write the solution as $f_{a i}^{(1)}(\vec{v})=n_{a i} \phi_{a}(v) h_{a i}(\vec{v})$ and express the left-hand side in terms of collision kernels. Summing the resulting equation over the charge-state indices, we have

$$
\begin{aligned}
\sum_{b} \int d^{3} v^{-} & {\left[\delta_{a b} J_{a}\left(\vec{v}, \vec{v}^{-}\right)+k_{a b}\left(\vec{v}, \vec{v}^{-}\right)\right] \bar{h}_{b}\left(\vec{v}^{-}\right) } \\
& =\left[\nabla_{\|} \ln p_{a}-\left(\frac{5}{2}-t_{a}\right) \nabla_{\|} \ln T_{a}\right] v_{\|{ }^{n} \phi_{a}}(v),
\end{aligned}
$$

where $n_{a}$ and $p_{a}$ are the total density and pressure of ion species $\underline{a}$ : 


$$
n_{a}=\frac{p_{a}}{T_{a}}=\sum_{i} n_{a 1} .
$$

Now Eq. (18) is just the kinetic equation for a system of electrons and simple ions, each ion species having a density $n_{a}$ and a charge $e_{a}$ defined by

$$
e_{a}^{2}=\frac{1}{n_{a}} \sum_{i} n_{a 1} e_{a i}^{2} .
$$

Note that these reduced densities and charges are different from those in the previous section.

In this reduced system, the parallel flows and heat fluxes, defined by

$$
\left(\begin{array}{c}
\bar{u}_{a} \\
\frac{2 \bar{q}_{a}}{5 n_{a} T_{a}}
\end{array}\right)=\int d^{3} v \phi_{a}(v) \bar{h}_{a}(\vec{v}) v_{1}\left(\begin{array}{c}
1 \\
t_{a}-\frac{5}{2}
\end{array}\right),
$$

can be related as follows to the parallel pressure and temperature gradients:

$$
\sum_{b}\left(\begin{array}{cc}
\bar{l}_{a b}^{11} & \bar{l}_{a b}^{12} \\
\bar{l}_{a b}^{21} & \bar{l}_{a b}^{22}
\end{array}\right)\left(\begin{array}{c}
\bar{u}_{b} \\
\frac{2 \bar{q}_{b}}{5 n_{b} T_{b}}
\end{array}\right)=\left(\begin{array}{c}
\nabla_{\| l} p_{a} \\
\frac{5}{2} n_{a} \nabla_{a} T_{a}
\end{array}\right) .
$$

The matrix of proportionality in the last equation contains the classical friction coefficients $\bar{l}_{\mathrm{ab}}^{\mathrm{kk}^{\prime}}$ of the reduced system. These are completely determined by the reduced system; expressions appropriate to the disparate mass limit, for example, can be found in Ref. 4. The information in Eq. (22) will be used in the calculation of the classical friction coefficients of the full system. 
Turning to the full system, we insert Eq. (18) into Eq. (17) and are left with an equation for $h_{a i}-\bar{h}_{a}$ :

$$
\begin{aligned}
\int d^{3} v^{-} J_{a}\left(\vec{v}, \vec{v}^{-}\right)\left[h_{a i}(\vec{v})-\bar{h}_{a}(\vec{v})\right] \\
=\left[\nabla_{n} p_{a i}-\xi_{a i} \nabla_{n} p_{a}-\left(\frac{5}{2}-t_{a}: n_{a i}-\xi_{a i} n_{a}\right) \nabla_{n} T_{a}\right] \frac{v_{n}}{\xi_{a i} T_{a}} \phi_{a}(v) .
\end{aligned}
$$

This equation involves the same test-particle kernel as was encountered in the previous section. In order to extract from it the classical friction coefficients of the full system, we first apply the operation $\int d^{3} v v_{\|} L_{k}^{(3 / 2)}\left(t_{a}\right)$. This converts it to the system of equations

$$
\begin{aligned}
\xi_{a i} \sum_{k^{\prime}=i}^{\infty} z_{a}^{k k^{-}}\left(u_{a i}^{k^{-}}-u_{a}^{-k^{-}}\right)= & \delta_{k 0}\left(\nabla_{n} p_{a i}-\xi_{a i} \nabla_{n} p_{a}\right) \\
& -\frac{5}{2} \delta_{k 1}\left(n_{a i}-\xi_{a i} n_{a}\right) \nabla_{l} T_{a},
\end{aligned}
$$

where the u's are Laguerre-polynomial moments of the distribution function:

$$
\begin{aligned}
& u_{a i}^{k}=\frac{3 \sqrt{\pi} k !}{4 \Gamma(k+5 / 2)} \int d^{3} v \phi_{a}(v) h_{a i}(\vec{v}) v_{n} L_{k}^{(3 / 2)}\left(t_{a}\right), \\
& u_{a}^{-k}=\sum_{I} \xi_{a i} u_{a i}^{k},
\end{aligned}
$$

and $Z_{a}^{\mathrm{kk}^{\circ}}$ is an appropriate matrix element of the test-particle kernel: 


$$
\begin{aligned}
Z_{a}^{k k^{-}} & =\frac{m_{a}^{2}}{3 T_{a}} \int d^{3} v^{3} d^{3} v^{-}\left(\vec{v}^{-} \cdot \vec{v}^{-}\right) L_{k}^{(3 / 2)}\left(t_{a}\right) J_{a}\left(\vec{v}, \vec{v}^{-}\right) L_{k}^{(3 / 2)}\left(t_{a}^{-}\right) \\
& =\frac{2 \pi m_{a} v_{T a}^{6}}{3} \int_{0}^{\infty} d t t^{1 / 2} \int_{0}^{\infty} d t^{-}\left(t^{-}\right)^{1 / 2} L_{k}^{(3 / 2)}(t) j_{a}\left(t, t^{-}\right) L_{k^{-}}^{(3 / 2)}\left(t^{-}\right),
\end{aligned}
$$

with $\tilde{j}_{a}$ as in Eq. (13). Within the context of the reduced system, $\mathrm{z}_{a}^{k k^{\prime}}$ is related to the matrix element $M_{a b}^{k^{-}}$introduced in Ref. 4 by $Z_{a}^{k^{-}}=m_{a} \sum_{b} M_{a b}^{k k^{-}}$. The first two moments in Eq. (25) give the parallel flow and the parallel heat flux, via

$$
u_{a i}^{0}=u_{a i}, \quad u_{a i}^{1}=-\frac{2 q_{a 1}}{5 n_{a i}{ }^{\top}} \text {. }
$$

Now we eliminate all moments except for the first two from Eq. (24), and combine the result with Eq. (22). We then end up with a relationship of the desired form, namely

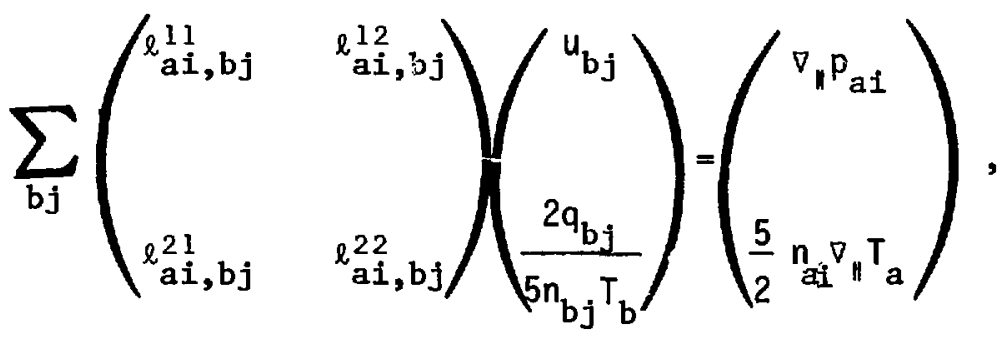

with classical friction coefficients given by

$$
e_{a i, b j}^{k k^{-}}=(-1)^{k+k k^{-}} \delta_{a b}\left(\delta_{i j} \xi_{a i}-\xi_{a i} \xi_{a j}\right) \hat{z}_{a}^{k-1, k^{-}-1}+\xi_{a i} \xi_{b j} \bar{l}_{a b}^{k k^{-}} .
$$

Here we have introduced the notation

$$
\hat{z}_{a}^{k k^{-}}=z_{a}^{k k^{-}}-\sum_{\ell, \ell^{\prime}=2}^{\infty} z_{a}^{k \ell}\left(\tilde{z}_{a}^{-1}\right)^{\ell \ell^{-}} z_{a}^{\ell^{\prime} k^{*}},
$$


where $\tilde{z}_{a}^{-1}$ is the inverse of $z_{a}^{l l^{\prime \prime}}$ on the space with $\ell \geq 2$ and $\ell^{-} \geq 2$ :

$$
\sum_{\ell^{-\infty}=2}^{\infty} z_{a}^{\ell \ell^{--}}\left(z_{a}^{-1}\right)^{\ell^{-\infty} \ell^{-}}=\delta_{\ell \ell^{-}}, \quad\left(\ell \geq 2, \ell^{-} \geq 2\right) .
$$

Equation (30) expresses the classical friction coefficients of the full system in terms of the classical friction coefficients and test-particle matrix elements of the reduced system. The same explicit charge-state dependence was obtained in the special model considered in Ref. 1. If in Eq. (30) the reduced system is considered in the disparate mass limit and the Laguerre expansion is truncated at $k=2$, we recover precisely the results of that model.

We now turn to the Pfirsch-Sch? üter radial transport coefficients. The specific results deperd on which particular temperatures are chosen to have equilibrated along the magnetic field line. We shall consider the case in which all the ions have equilibrated to a temperature $T$ and the electrons to a temperature $T_{e}$. The form of the transport equations is then as noted, for example, in Ref. 1. The radial particle and heat fluxes associated with the electrons are given by

$$
\begin{aligned}
& r_{e}^{P S}=\frac{1}{e} G_{1}\left[l_{e e_{n_{e}}^{11}}^{p_{e}^{-}}+l_{e e}^{12} \frac{T_{e}^{-}}{e}-\sum_{a i} l_{e, a i}^{11} \frac{p_{a i}^{-}}{n_{a i} e_{a i}}\right], \\
& q_{e}^{P S}=G_{1} \frac{T_{e}}{e}\left[l_{e e}^{21} \frac{p_{e}^{-}}{n_{e}^{e}}+l_{e e}^{22} \frac{T_{e}^{e}}{e}-\sum_{a i} \ell_{e, a i}^{21} \frac{p_{a i}^{-}}{n_{a i} e_{a i}}\right],
\end{aligned}
$$

where $G_{1}$ is the Pfirsch-Schlüter geometrical factor $F(\psi)^{2}\left(\left\langle B^{-2}\right\rangle-\left\langle B^{2}\right\rangle^{-1}\right)$ and the primes denote $\partial / \partial \psi$. Beginning with the last two equations, the electrons are labeled explicitly and indices such as (ai) refer only to the ions. Since 
the electrons are always disparate in mass with respect to the ions, the particular expressions for $e_{e e}^{k^{\prime}}$ and $\ell_{e, a i}^{k k^{\prime}}$ obtained previously [Ref. 1, Eqs. (6.12)] continue to apply.

The ion pa:ticle flux and heat flux (the latter summed over all ions) are given by the nore complicated expressions

$$
\begin{aligned}
r_{a i}^{P S}= & \frac{1}{e_{a i}} G_{1}\left[-l_{a i, e}^{11} \frac{p_{e}^{-}}{n_{e}^{e}}-l_{a i, e}^{12} \frac{T_{e}^{-}}{e}+\frac{1}{L} S L_{a i} T^{\circ}\right. \\
& \left.+\sum_{b j}\left(L_{a i, b j}+\frac{1}{L} L_{a i} L_{b j}\right) \frac{p_{b j}^{-}}{n_{b j} e_{b j}}\right], \\
q^{P S}= & G_{1} \frac{S T}{L}\left[\sum_{a i} L_{a i} \frac{p_{a i}^{-}}{n_{a i} e_{a i}}+S T^{-}\right],
\end{aligned}
$$

where $s=\sum_{a i}\left(n_{a i} / e_{a i}\right)$. The ion radial transport coefficients, as represented by the quantities $L_{a i, b j}, L_{a i}$, and $L$, are related to the classical friction coefficients by general expressions noted in Ref. 1. Using Eq. (30) to evaluate them, we firally obtain

$$
\begin{aligned}
& L_{a i, b j}=\delta_{a b}\left(\delta_{i j} \xi_{a i}-\xi_{a i} \xi_{b j}\right)\left[\hat{z}_{a}^{00}-\frac{\left(\hat{Z}_{a}^{01}\right)^{2}}{\hat{z}_{a}^{11}}\right]
\end{aligned}
$$

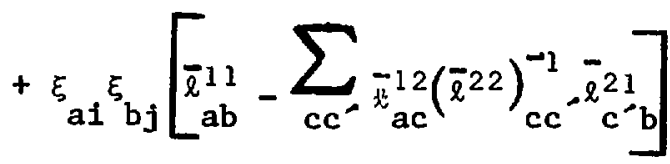

$$
\begin{aligned}
& -\delta_{a b} \delta_{i j} m_{e} n_{e} e_{e e} \frac{n_{a i} e_{a i}^{2}}{n_{e} e^{2}}+m_{e} e_{e} e_{e e} \frac{n_{a i} e_{a i}^{2} n_{b j} e_{b j}^{2}}{\left(n_{e} e^{2}\right)^{2} e_{e}}\left(1-c_{1}^{e}\right),
\end{aligned}
$$




$$
\begin{aligned}
L_{a i} & =-\left(n_{a i}-\xi_{a i} n_{a}\right) \frac{\hat{Z}_{a}^{01}}{\hat{Z}_{a}^{11}}+\xi_{a i} \sum_{b c} \bar{l}_{a b}^{12}\left(\bar{l}^{22}\right)_{b c}^{-1} n_{c}, \\
L & =\sum_{a} \frac{n_{a}^{2} \psi_{a}}{\hat{Z}_{a}^{11}}+\sum_{a b} n_{a}\left(\bar{l}^{22}\right)_{a b}^{-1} n_{b},
\end{aligned}
$$

where $\psi_{a}$ is the following average of the charge distribution within ion species $\underline{\text { a }}:$

$$
\psi_{a}=\frac{1}{2 n_{a}^{2}} \sum_{i j} n_{a i} n_{a j}\left(\frac{e_{a i}}{e_{a j}}-\frac{e_{a j}}{e_{a i}}\right)^{2},
$$

and $c_{l}^{e}$ is a function $(1)$ of the effective ionic charge $z_{e}$. It is to be recalled that the indices $a, b$, etc., in these equations refer only to the ions, since now the electrons are labeled separately. The system in which the quantities $\hat{Z}_{a}$ and $\bar{l}_{a b}$ are to be evaluated contains only reduced ions (and not electrons). As one would expect, these results reduce to those of Ref. 1 when the disparate mass limit and truncated Laguerre expansion are employed.

\section{ACKNOWLEDGMENT}

The author would like to thank E. M. Gelbard for useful discussions. 


\section{REFERENCES}

1. C. D. BOLEY, E. M. GELBARD and S. P. HIRSHMAN, "Disparate Clump Approximation in Neoclassical Plasma Transport Theory," ANL/FPP/TM-113 (1978).

2. Errata for Ref. 1: In Eq. (6.1) reverse the sign of the term containing $\nabla_{\|} T_{a}$; in the third equation of (6.12), read $C_{2}^{e}$ for $c^{e}$.

3. S. P. HIRSHMAN, D. J. SIGMAR and J. F. CLARKE, Phys. Fluids 19, 656 (1976);

S. P. HIRSHMAN and D. J. SIGMAR, Phys. F'Zuids 20, 418 (1977); S. P. HIRSHMAN Phys. Fluids 21, 1295 (1978).

4. S. P. HIRSHMAN, Phys. Fluids 20, 589 (1977).

5. The radial transport coefficients also have a "classical" contribution (written out, for example, in Ref. 1), which is trivial from a computational point of view. 


\section{Internal:}

P. Amundson

W. P. Barthold

M. Benson

P. B. Bertoncini

C. D. Boley

J. N. Brooks

Y. Chang

D. A. Ehst

E. Evans, Jr.

E. M. Gelbard

M. Y. Gohar
I. R. Greenwood

D. M. Gruen

J. Jung

H. Henryson

M. Kaminsky

R. Kustom

L. LeSage

V. Maroni

J. Norem

F. Nolfi

P. J. Persiani
A. B. Smith

W. J. Sturm

C. E. Ti11

A. Trave11i

D. C. Wade

C. Hytry (20)

FP Program (2)

A. B. Krisciunas

ANL Contract File

ANL Libraries (5)

TIS Files (6)

\section{Externa1:}

DOE-TIC, for distribution per UC-20a and -20d (214)

Manager, Chicago Operations office

Chief, Office of Patent Counsel, $\mathrm{CH}$

President, Argonne Universities Association

Applied Physics Division Review Committee

P. W. Dickson, Jr., Westinghouse Electric Corp.

R. L. Hellens, Combustion Engineering, Inc.

W. B. Loewenstein, Electric Power Research Institute

R. F. Redmond, Ohio State University

R. Sher, Stanford University

D. B. Wehmeyer, Detroit Edison Co.

S. A. Werner, University of Missouri

M. A. Abdou, Georgia Institute of Technology

E. C. Crume, Oak Ridge National Laboratory

S. P. Hirshman, Oak Ridge National Laboratory

J. T. Hogan, Oak Ridge National Laboratory

F. L. Hinton, University of Texas, Austin

W. W. Pfeiffer, General Atomic

D. E. Post, Princeton Plasma Physics Laboratory

J. N. Davidson, Georgia Institute of Technology

W. M. Stacey, Jr., Georgia Institute of Technology

T. Uckan, Oak Ridge National Laboratory

C. E. Wagner, Science Applications, Inc.

R. E. Waltz, General Atomic 